\title{
Effects of Dezocine-Remifentanil Intravenous Anaesthesia on Perioperative Signs, Serum TNF- $\alpha$ and IL-6 in Liver Cancer Patients undergoing Radiofrequency Ablation
}

\author{
Qiang Jia1, Fen Tian², Wei-Na Duan³, Yi-Fan Jia1, Hua-Xin Wang³ and Zhong-Yuan Xia3
}

\begin{abstract}
Objective: To analyse the impact of dezocine-remifentanil intravenous anaesthesia on perioperative signs, serum tumour necrosis factor- $\alpha$ (TNF- $\alpha$ ), and interleukin-6 (IL-6) in liver cancer patients undergoing radiofrequency ablation (RFA).

Study Design: An experimental study.

Place and Duration of Study: Renmin Hospital of Wuhan University, Wuhan, China, from January 2017 to February 2018.

Methodology: Eighty patients with small hepatocellular carcinoma (SHCC) were selected as the research object. They were divided into Group A and Group B with the random number table method, with 40 cases in each group. Group A were given dezocine-remifentanil intravenous anaesthesia and Group B were given midazolam-remifentanil intravenous anaesthesia. Patients' situations in the surgery were compared between the two groups. Changes in heart rate (HR), mean arterial pressure (MAP) and blood oxygen saturation $\left(\mathrm{SpO}_{2}\right)$ were recorded before the surgery $\left(\mathrm{T}_{0}\right)$, at 5 minutes after the RFA $\left(T_{1}\right)$ and at the end of the RFA $\left(T_{2}\right)$. Levels of tumour necrosis factor- $\alpha$ (TNF- $\alpha$ ) and interleukin-6 (IL-6) on the $1^{\text {st }}$ day after the RFA were compared between the two groups.

Results: The wake-up time in Group A was shorter than Group B $(p<0.001)$, and the VAS pain score in Group A was lower than Group B $(p<0.001)$. At $T_{1}$, the MAP in Group A was higher than Group B $(p<0.001)$. There was no significant difference in MAP between the two groups at $T_{0}$ and $T_{2}\left(p=0.881,0.696\right.$, respectively). At $T_{1}$ and $T_{2}$, the HR in Group $A$ was lower than Group B (all $p<0.001)$. There was no significant difference in HR between the two groups at $T_{0}(p=0.684)$. There was no significant difference in $\mathrm{SpO}_{2}$ between the two groups at $\mathrm{T}_{0}, \mathrm{~T}_{1}$ and $\mathrm{T}_{2}(\mathrm{p}=0.654,0.884$ and 0.798 , respectively). On the 1st day after the RFA, the level of TNF- $\alpha$, IL-6 in Group A were lower than those of Group B (all $p<0.001$ ). There was no significant difference in the incidence of intraoperative complications between the two groups $(p=0.644)$.

Conclusion: Compared with midazolam-remifentanil intravenous anaesthesia, the dezocine-remifentanil method has a better analgesic effect, shorter wake-up time, and can effectively regulate the expression of inflammatory cytokines TNF- $\alpha$ and IL-6. However, the effect of remifentanil on the respiratory function is dose-dependent. Therefore, respiratory cycle monitoring and management should be strengthened during the surgery.
\end{abstract}

Key Words: Dezocine, Midazolam, Remifentanil, Liver tumours, Radiofrequency ablation, Tumour necrosis factor- $\alpha$, Interleukin-6.

\section{INTRODUCTION}

Radiofrequency ablation (RFA) is currently considered to be an effective minimally invasive treatment for small hepatocellular carcinoma. It has the characteristics of less trauma and faster postoperative recovery. It is the latest method for the treatment of primary and metastatic liver cancers.1,2 However, RFA often has a short operative time with strong intraoperative stimulation but less than resection, so general local anaesthesia or a single use of analgesic drugs cannot achieve satisfactory results. ${ }^{3}$ To improve the comfort of patients

Department of Pain 1 / Operating Room² / Anesthesiology3,

Renmin Hospital of Wuhan University, Wuhan, 430060, China

Correspondence: Dr. Zhong-Yuan Xia, Department of

Anesthesiology, Renmin Hospital of Wuhan University,

Wuhan, ,430060, China

E-mail:am8dr6e5@sina.com

Received: April 19, 2018; Accepted: September 09, 2018 during the RFA treatment and raise the thoroughness of the RFA treatment, intravenous anaesthesia can be used. Since remifentanil works faster and has small impacts on hemodynamics and hormone levels, it is often used for intravenous anaesthesia. However, the large dosage of remifentanil can easily cause respiratory depression.4,5 Dezocine is a synthetic drug of the benzmorphan group, an agonist of $\kappa$-opioid receptors and an antagonist of $\mu$-opioid receptors. It has moderate analgesic and sedative effects and is very safe without respiratory depression. ${ }^{6}$ Under high temperature, RFA is prone to cause endotoxemia and releases cytokines such as interleukin-6 (IL-6) and tumour necrosis factor- $\alpha$ (TNF- $\alpha$ ), which will aggravate the inflammatory reaction and cause tissue damage. ${ }^{7}$ Currently, the effects of dezocine-remifentanil intravenous anaesthesia on perioperative signs, serum TNF- $\alpha$ and IL-6 in liver cancer patients undergoing radiofrequency ablation (RFA) remain unclear. 
This study was conducted to determine the anaesthetic effect and safety of dezocine combined with remifentanil and the impact on inflammatory factors in the RFA surgery, with a view to provide a reference for the anaesthetic management in the radiofrequency ablation of liver cancer.

\section{METHODOLOGY}

This study was conducted at the Renmin Hospital of Wuhan University, Wuhan, China, from January 2017 to February 2018. Eighty patients with small hepatocellular carcinoma, who underwent RFA, were selected as the research object. Inclusion criteria were patients who had RFA indications, no cardiovascular diseases, severe liver and kidney dysfunction, and had consented for the procedure. Exclusion criteria were patients with central nervous and mental diseases, history of long-term use of opioids, acute and chronic infections and patients taking non-steroidal anti-inflammatory drugs (NSAIDs) or hormones.

The study was approved by the Hospital Ethical and Research Committee, and all patients signed the informed consents. Eighty patients were divided into Group A and Group B by random number table method, with 40 cases in each group. Group A were given dezocine-remifentanil intravenous anaesthesia and Group B were given midazolam-remifentanil intravenous anaesthesia.

The two groups of patients were given regular fasting and no drinking before the surgery. Intraoperative detection of heart rate (HR), mean arterial pressure (MAP) and blood oxygen saturation $\left(\mathrm{SpO}_{2}\right)$ were performed during the surgery. The nasal oxygen inhalation were given at an oxygen flow of $2 \mathrm{~L} /$ minute. Group A: intravenous dezocine, loading dose $0.8 \mathrm{mg} / \mathrm{kg}$. Group B: intravenous midazolam, loading dose 0.05 0.1 $\mathrm{mg} / \mathrm{kg}$. The loading doses in both groups were diluted to $20 \mathrm{ml}$ with $0.9 \%$ sodium chloride solution and the injection time was $10 \mathrm{~min}$. At 5 minutes after injection, intravenous remifentanil at a loading dose of $0.2 \mu \mathrm{g} / \mathrm{Kg}$ was given. After the start of the surgery, remifentanil was pumped at $0.05 \mu \mathrm{g} /(\mathrm{Kg} /$ minute $)$. The speed of remifentanil administration was adjusted according to hemodynamics. If patients showed hypotension [BP $90 / 60 \mathrm{mmHg}(1 \mathrm{mmHg}=0.133 \mathrm{KPa})]$, ephedrine would be used to boost the blood pressure. If patients showed hypertension ( $B P \geq 140 / 90 \mathrm{mmHg}$ ), urapidil would be given to reduce the blood pressure. If patients showed bradycardia (HR $\leq 60$ times/min), atropine would be immediately used. For tachycardia (HR $\geq 100$ times/min), esmolol would be immediately used. For respiratory depression $\left(\mathrm{SpO}_{2}<95 \%\right)$, mask-assisted ventilation would be given and mask-pressurised ventilation would also be selected when necessary. Anaesthesia was discontinued in both groups at the end of the surgery.
The postoperative wake-up time was recorded in both groups and the visual analogue scale (VAS) was used to evaluate the degree of pain in patients. The score ranged from 0 to 10 points. The higher the score was, the severer the pain would be. Changes of HR, MAP and $\mathrm{SpO}_{2}$ before the surgery $\left(\mathrm{T}_{0}\right)$, at 5 min after the RFA $\left(\mathrm{T}_{1}\right)$ and at the end of the RFA $\left(T_{2}\right)$ were recorded. The levels of TNF- $\alpha$ and IL- 6 on the 1st day after the RFA were measured with the double antibody sandwich enzymelinked immunosorbent assay (ELISA). The incidences of complications such as respiratory depression, hypotension, nausea and vomiting in the two groups during the surgery were recorded.

SPSS 21.0 statistical software was used for data analysis. Count data were expressed in $n(\%)$ and the $x^{2}$ test was performed. Measurement data were expressed as mean $\pm S D$. The independent samples t-test was used for comparison between the groups. A difference with a $p$-value less than 0.05 was considered statistically significant.

\section{RESULTS}

Among the 80 patients, $41(51.25 \%)$ were males and 39 $(48.75 \%)$ were females; the age ranged from 29 to 67 $(52.73 \pm 3.18)$ years; tumour diameter $1 \sim 5(3.24 \pm 0.58)$ $\mathrm{cm}$; body mass 44 72 (60.36 \pm 2.43$) \mathrm{kg}$; ASA Grade I 37 cases (46.25\%), ASA Grade II 43 cases (53.75\%).

The wake-up time in Group A was shorter than Group B $(p<0.001)$, and the VAS pain score in Group A was lower than Group B $(p<0.001$, Table I).

At $T_{1}$, the MAP in Group A was higher than Group B $(p<0.001)$. There was no significant difference in MAP between the two groups at $T_{0}$ and $T_{2}(p=0.881,0.696$, respectively). At $T_{1}$ and $T_{2}$, the $H R$ in Group $A$ was lower than Group B (all $p<0.001)$. There was no significant difference in $H R$ between the two groups at $T_{0}$ $(p=0.684)$. There was no significant difference in $\mathrm{SpO}_{2}$ between the two groups at $\mathrm{T}_{0}, \mathrm{~T}_{1}$ and $\mathrm{T}_{2}(\mathrm{p}=0.654,0.884$ and 0.798 , respectively, Table II). On the first day after the RFA, the level of TNF- $\alpha$ IL- 6 in Group A were lower than those of Group B (all $p<0.001$, Table III).

The incidence of intraoperative complications in Group A was $5.00 \%$ (2 cases), i.e. mild respiratory depression, and nausea and vomiting in one case each. The incidence of intraoperative complications in Group B was $7.50 \%(n=3)$, i.e. mild respiratory depression, hypotension, and nausea and vomiting in one case each. There was no significant difference in the

Table I: Comparison of wake-up time and VAS pain scores in the two groups.

\begin{tabular}{l|c|cc|cc}
\hline \multirow{2}{*}{ Group } & $\mathrm{n}$ & \multicolumn{2}{|c|}{ Wake-up time (min) } & \multicolumn{2}{c}{ VAS pain scores (score) } \\
\cline { 3 - 6 } & & Mean \pm SD & p-value & Mean \pm SD & p-value \\
\hline Group A & 40 & $5.34 \pm 0.68$ & $<0.001$ & $1.41 \pm 0.46$ & $<0.001$ \\
Group B & 40 & $9.02 \pm 1.01$ & & $3.95 \pm 0.56$ & \\
\hline
\end{tabular}


Table II: Comparison of MAP, $\mathrm{HR}$ and $\mathrm{SpO}_{2}$ in the two groups at different time points.

\begin{tabular}{|c|c|c|c|c|c|c|c|c|}
\hline \multirow[t]{2}{*}{ Index } & \multirow[t]{2}{*}{ Group } & \multirow[t]{2}{*}{$\mathrm{n}$} & \multicolumn{2}{|c|}{$\mathrm{T}_{0}$} & \multicolumn{2}{|c|}{$\mathrm{T}_{1}$} & \multicolumn{2}{|c|}{$\mathrm{T}_{2}$} \\
\hline & & & Mean \pm SD & $p$-value & Mean \pm SD & p-value & Mean \pm SD & $\mathrm{p}$-value \\
\hline \multirow[t]{2}{*}{ MAP (mmHg) } & Group A & 40 & $95.02 \pm 5.99$ & \multirow[t]{2}{*}{0.881} & $81.45 \pm 3.54$ & \multirow[t]{2}{*}{$<0.001$} & $83.17 \pm 4.05$ & \multirow[t]{2}{*}{0.696} \\
\hline & Group B & 40 & $94.86 \pm 3.04$ & & $72.62 \pm 4.56$ & & $82.75 \pm 5.43$ & \\
\hline \multirow[t]{2}{*}{ HR (frequency/min) } & Group A & 40 & $88.59 \pm 3.84$ & \multirow[t]{2}{*}{0.684} & $63.08 \pm 5.57$ & \multirow[t]{2}{*}{$<0.001$} & $76.26 \pm 7.09$ & \multirow[t]{2}{*}{$<0.001$} \\
\hline & Group B & 40 & $89.01 \pm 5.26$ & & $72.16 \pm 6.08$ & & $83.04 \pm 7.60$ & \\
\hline \multirow[t]{2}{*}{$\mathrm{SpO}_{2}(\%)$} & Group A & 40 & $98.04 \pm 1.01$ & \multirow[t]{2}{*}{0.654} & $93.28 \pm 1.62$ & \multirow[t]{2}{*}{0.884} & $93.91 \pm 1.27$ & \multirow[t]{2}{*}{0.798} \\
\hline & Group B & 40 & $98.17 \pm 1.52$ & & $93.34 \pm 2.03$ & & $94.02 \pm 2.40$ & \\
\hline
\end{tabular}

Table III: Comparison of TNF- $\alpha$ and IL-6 levels in the two groups on the 1 st day after the RFA.

\begin{tabular}{|c|c|c|c|c|c|}
\hline \multirow[t]{2}{*}{ Group } & \multirow[t]{2}{*}{$n$} & \multicolumn{2}{|c|}{ TNF- $\alpha(p g / m L)$} & \multicolumn{2}{|c|}{ IL-6 (pg/mL) } \\
\hline & & Mean \pm SD & $\mathrm{p}$-value & Mean \pm SD & $\mathrm{p}$-value \\
\hline Group A & 40 & $31.74 \pm 5.72$ & $<0.001$ & $103.41 \pm 15.19$ & $<0.001$ \\
\hline Group B & 40 & $43.66 \pm 5.40$ & & $152.38 \pm 25.32$ & \\
\hline
\end{tabular}

incidence of intraoperative complications between the two groups $(p=0.644)$.

\section{DISCUSSION}

Radiofrequency ablation for liver cancer is a minimally invasive operation that can effectively treat small hepatocellular carcinoma. The radiofrequency electrode needle can raise the local centre temperature to $90^{\circ} \mathrm{C}$ or above, resulting in the solidification and necrosis of liver cancer tissues and thus killing cancer cells.8,9 Radiofrequency ablation for liver cancer does not require muscle relaxation and the operative time is short. However, the patient usually has a high fever, abdominal pain and other discomforts, so it is necessary to maintain a moderate degree of sedation and analgesia, and intravenous general anaesthesia without tracheal intubation is always selected.

Remifentanil, as a new-type ultrashort opioid analgesic agent, works fast and can be quickly metabolised, without drug accumulation. In clinical practice, the dosage is highly controllable as the infusion speed can be adjusted according to the patient's intraoperative blood pressure, $\mathrm{SpO}_{2}$, etc. ${ }^{10,11}$ In addition, remifentanil is mainly metabolised by non-specific hydrolysis in blood and tissues, and a small amount of unmetabolised drugs can be antagonised by opioid receptor antagonists without affecting liver and kidney function. 12,13

Dezocine has a stronger analgesic effect than other opioids. ${ }^{14}$ In addition, dezocine can relax the gastrointestinal smooth muscle, reduce the occurrence of adverse reactions such as nausea and vomiting, and has few adverse reactions to respiratory depression, and there is generally no significant respiratory depression at therapeutic doses. Dezocine also has a sedative effect and the patient can be awakened, which can help with the patient's short breath holding action required by the RFA for liver cancer, and also can achieve moderate sedation and analgesia. It is an ideal intraoperative and postoperative intravenous analgesic agent. ${ }^{15-17}$

The results of this study showed that there were significant differences between the two groups in postoperative wake-up time and VAS pain scores. The decrease of $H R$ at $T_{1}$ and $T_{2}$ in Group $A$ was significant and obviously lower than that in Group B. The reason may be that the effect of remifentanil on the respiratory function was dose-dependent, and the increased dose may cause respiratory depression. In addition, because some elderly patients had decreased respiratory and drug metabolism functions, the risk of respiratory depression during anaesthesia increased. ${ }^{18}$ There was no significant difference in the incidence of intraoperative complications between the two groups, but mild respiratory depression occurred in two cases in this study. Therefore, clinical attention should be paid to the dynamic monitoring of the respiratory circulation. The speed of remifentanil infusion should be adjusted according to the changes of HR to improve the safety of the surgery.

TNF- $\alpha$ and IL- 6 are important pro-inflammatory factors, in which TNF- $\alpha$ can activate endothelial cells, cause neutrophil aggregation, promote the release of IL- 6 and other inflammatory factors. IL- 6 can be transformed into hepatocyte activating factors under stress conditions and induce the production of acute-phase reactive proteins. ${ }^{19,20}$ Remifentanil can reduce the concentration of serum TNF- $\alpha$ and decrease the inflammatory level of the body. Dezocine can regulate the level of cytokines during the perioperative period of liver cancer RFA and reduce tumour angiogenesis. ${ }^{21}$ The results of this study showed that the levels of TNF- $\alpha$ and IL-6 in Group A after the RFA were lower than those in Group B, suggesting that dezocine-remifentanil intravenous anaesthesia had more advantages in regulating the release of cytokines.

\section{CONCLUSION}

Compared with midazolam-remifentanil intravenous anaesthesia, the dezocine-remifentanil method has a better analgesic effect, shorter wake-up time, and can effectively regulate the expression of inflammatory cytokines TNF- $\alpha$ and IL-6. However, the effect of remifentanil on the respiratory function is dose- 
dependent. Therefore, respiratory cycle monitoring and management should be strengthened during the surgery.

\section{REFERENCES}

1. Kuck KH, Brugadan J, Fürnkranz A, Metzner A, Ouyang F, Chun KR, et al. Cryoballoon or radiofrequency ablation for paroxysmal atrial fibrillation. N Engl J Med 2016; 374:2235-45.

2. Lencioni R, Crocetti L. Radiofrequency ablation of liver cancer. Tech Vasc Interv Radiol 2007; 10:38-46.

3. Lai R, Peng Z, Chen D, Wang X, Xing W, Zeng W, et al. The effects of anesthetic technique on cancer recurrence in percutaneous radiofrequency ablation of small hepatocellular carcinoma. Anesth Analg 2012; 114:290-6.

4. Kuo YH, Chung KC, Hung $\mathrm{CH}$, Lu SN, Wang JH. The impact of general anesthesia on radiofrequency ablation of hepatocellular carcinoma. Kaohsiung J Med Sci 2014; 30:559-65.

5. Hogue CW Jr, Bowdle TA, O'Leary C, Duncalf D, Miguel R, Pitts $M$, et al. A multicenter evaluation of total intravenous anesthesia with remifentanil and propofol for elective inpatient surgery. Anesth Analg 1996; 83:279-85.

6. Martorano PP, Aloj F, Baietta S, Fiorelli A, Munari M, Paccagnella $\mathrm{F}$, et al. Sufentanil-propofol versus remifentanilpropofol during total intravenous anesthesia for neurosurgery. a multicentre study. Minerva Anestesiol 2008; 74:233-43.

7. Shannon E, Noveck R, Sandoval F, Kamath B, Kearney M. Thalidomide suppressed interleukin- 6 but not tumor necrosis factor-alpha in volunteers with experimental endotoxemia. Transl Res 2007; 150:275-80.

8. Kondo Y, Yoshida H, Shiina S, Tateishi R, Teratani T, Omata M. Artificial ascites technique for percutaneous radiofrequency ablation of liver cancer adjacent to the gastrointestinal tract. Br J Surg 2006; 93:1277-82.

9. Kondo $Y$, Yoshida H, Tateishi R, Shiina S, Kawabe T, Omata M. Percutaneous radiofrequency ablation of liver cancer in the hepatic dome using the intrapleural fluid infusion technique. Br J Surg 2008; 95:996-1004.

10. Park SW, Yi JW, Kim YM, Kang JM, KIm DO, Shin MS, et al. Remifentanil alleviates transient cerebral ischemia-induced memory impairment through suppression of apoptotic neuronal cell death in gerbils. Korean J Anesthesiol 2011; 61:63-8.

11. Poon RT, Borys N. Lyso-thermosensitive liposomal doxorubicin: an adjuvant to increase the cure rate of radiofrequency ablation in liver cancer. Future Oncol 2011; 7:937-45.

12. Lauwers M, Camu F, Vanlersberghe C. Remifentanil, an esterase-metabolised opioid. CNS Drugs 1997; 8:189-98.

13. Baraka AS, Haroun-Bizri ST, Nawfal MF, Gerges FJ, Nasr VG. Does pancuronium or cisatracurium delay the rate of arousal following remifentanil-based anesthesia? Middle East $J$ Anaesthesiol 2005; 18:477-84.

14. Zheng M, Guo Y, Shan S, Yang S. Dezocine for anesthesia and stress reduction in induced abortion. Patient Prefer Adherence 2015; 9:369-72.

15. Liu XS, Xu GH, Shen QY, Zhao Q, Cheng XQ, Zhang J, et al. Dezocine prevents sufentanil-induced cough during general anesthesia induction: a randomized controlled trial. Pharmacol Rep 2015; 67:52-5.

16. Tian ZY, Li HT, Liang GH, Piao CH, Quan SY, Cui WY, et al. Comparison of sufentanil and dezocine anesthetic effects on trigeminal neuralgia undergoing radiofrequency thermocoagulation. Chin J New Drugs 2014; 23:1171-4.

17. Li LJ. Progress in clinical application of artificial liver support systems. Zhonghna Gan Zang Bing Za Zhi 2005; 13:844-5.

18. Barbour SJ, Vandebeek CA, Ansermino JM. Increased tidal volume variability in children is a better marker of opioidinduced respiratory depression than decreased respiratory rate. J Clin Monit Comput 2004; 18:171-8.

19. Rochfort KD, Collins LE, Mcloughlin A, Cummins PM.TNF- $\alpha-$ mediated disruption of cerebrovascular endothelial barrier integrity in vitro involves the production of proinflammatory IL-6. J Neurochem 2016; 136:564-72.

20. Lin RY, Trivino MR, Curry A, Pesola GR, Knight RJ, Lee HS, et al. Interleukin-6 and c-reactive protein levels in patients with acute allergic reactions: an emergency department-based study. Ann Allergy Asthma Immunol 2001; 87:412-6.

21. Liu YH, Fan ZY, Jin YY. Efficiency and safety of dezocine in radiofrequency ablation for hepatic carcinoma. World Chin $\mathrm{J}$ Digestol 2015; 23:1165-9. 